\title{
2-D Modeling of Energy-z Beam Dynamics Using the LiTrack Matlab Program
}

\author{
Sean K. Cauley \\ Office of Science, SULI Program \\ Paine College \\ Stanford Linear Acceleration Center \\ Menlo Park, California
}

August 19, 2005

Prepared in partial fulfillment of the requirements of the Office of Science, U.S. Department of Energy Science Undergraduate Laboratory Internship (SULI) Program under the direction of Michael Woods in the Particle Physics Division of Stanford Linear Acceleration Center (SLAC).

Participant:

Signature

Research Advisor:

Signature 


\section{Table of Contents}

$\begin{array}{lr}\text { Abstract } & \text { ii } \\ \text { Introduction } & 1 \\ \text { Material and Methods } & 3 \\ \text { Results } & 6 \\ \text { Discussions and Conclusions } & 8 \\ \text { Acknowledgement } & 9 \\ \text { References } & 9 \\ \text { Tables } & 10\end{array}$

$\begin{array}{ll}\text { Figures } & 13\end{array}$ 


\begin{abstract}
2-D Modeling of Energy-z Beam Dynamics Using the LiTrack Matlab Program. SEAN K. CAULEY (Paine College, Augusta, GA 30901) MICHAEL WOODS (Stanford Linear Acceleration Center, Menlo Park, CA 94025).
\end{abstract}

Short bunches and the bunch length distribution have important consequences for both the LCLS project at SLAC and the proposed ILC project. For both these projects, it is important to simulate what bunch length distributions are expected and then to perform actual measurements. The goal of the research is to determine the sensitivity of the bunch length distribution to accelerator phase and voltage. This then indicates the level of control and stability that is needed. In this project I simulated beamlines to find the rms bunch length in three different beam lines at SLAC, which are the test beam to End Station A (ILC-ESA) for the ILC studies, Linac Coherent Light Source (LCLS) and LCLS-ESA. To simulate the beamlines, I used the LiTrack program, which does a 2-dimensional tracking of an electron bunch’s longitudinal (z) and the energy spread beam ( E) parameters. In order to reduce the time of processing the information, I developed a small program to loop over adjustable machine parameters. LiTrack is a Matlab script and Matlab is also used for plotting and saving and loading files. The results show that the LCLS in Linac-A is the most sensitive when looking at the ratio of change in phase degree to rate of change. The results also show a noticeable difference between the LCLS and LCLS-ESA, which suggest that further testing should go into looking the Beam Switch Yard and End Station A to determine why the result of the LCLS and LCLS-ESA vary. 


\section{INTRODUCTION}

The electron bunch distribution generated by the linear accelerator (linac) at Stanford Linear Accelerator Center (SLAC) can be described by a 6-parameter "phase space” $\left(\mathrm{x}, \mathrm{x}^{\prime}, \mathrm{y}, \mathrm{y}^{\prime}, \mathrm{z}, \mathrm{E} / \mathrm{E}\right)$. The $\mathrm{X}$ coordinate represents the horizontal transverse distribution with characteristic rms spotsize $\quad(\mathrm{x}) ; \mathrm{X}^{\prime}$ is the horizontal angular distribution with characteristic rms angular divergence $\left(\mathrm{x}^{\prime}\right)$. The $\mathrm{Y}$ coordinate represents vertical transverse distribution with characteristic rms spotsize $\quad(y)$; $Y^{\prime}$ is the vertical angular distribution with characteristic rms angular divergence (y'). $\mathrm{Z}$ is the longitudinal distribution with characteristic rms bunch length

(z). The sixth coordinate in the 6-parameter "phase space" is E/E. E/E represents the energy spread of the beam. The fifth and sixth coordinate ( $Z$ and $\quad E / E)$ determine the beam's longitudinal emittance, which is given by the product of the bunch length and energy spread distributions. In this project, I am studying the longitudinal emittance of electron bunch distributions for 3 planned beamlines at SLAC.

The three beamlines that will be use to analyze the longitudinal emittance of electron bunch distribution are i) Linac Coherent Light Source (LCLS), with beam from a new rf gun at the 2/3 point of the SLAC Linac to the end of the Linac, ii) LCLS-ESA, with the beam in i) transported through the A-line to End Station A, and iii) International Linear Collider (ILC) test beam using the full existing SLAC Linac and Damping Rings with beam transported from the end of the Linac through the A-line to End Station A (ILC-ESA) (see Figure 1).

For the LCLS beam study, we track the beam in the last third of the linac; it has a beam energy of $14.1 \mathrm{GeV}$ at the end of the Linac (see Figure 2). The LCLS project will use the 14.1 $\mathrm{GeV}$ electron beam and pass it through a new 100-meter wiggler section to be constructed to generate an intense short pulse $\mathrm{x}$-ray laser. Short electron bunches enable short $\mathrm{x}$-ray pulses. 
For the ILC-ESA, we tracked the beam from the existing electron damping ring through the full linac and to ESA, with a final beam energy of $28.5 \mathrm{GeV}$. This test beam has similar bunch charges and bunch lengths as will be used in the proposed ILC project.

The LCLS-ESA beamline adds the A-line to the LCLS beamline, with a final beam energy of $14.1 \mathrm{GeV}$.. This last beamline simulation will be to study how an LCLS test beam could replace the ILC-ESA beam for ILC tests.

Short bunches and the bunch length distribution have important consequences for both the LCLS project at SLAC and the proposed ILC project. For both these projects, it is important to simulate what bunch length distributions are expected and then to perform actual measurements. Here I report on simulation studies. At ILC, intense short bunches generate wakefields. Wakefields arise from image charges generated in material (collimators, beampipes) close to the electron bunch [1]. The image charges generate electromagnetic wakefields that act back on the bunch, in particular on the tail of the bunch, increasing both the transverse and longitudinal beam emittance [2]. These wakefields can cause a change in the longitudinal energy distribution of a bunch. Wakefields generated by short bunches can also escape from the beampipe (from ports for cables or vacuum pumps for example) and generate electromagnetic interference to detector electronics (2). At LCLS, short bunches will be used to see real time chemical and biological reactions [3]. Chemical and biological reaction occur at sub-picosecond time scale; therefore, it is important to generate bunches which are equivalent to $300 \mathrm{~m}$ or 1 picoseconds. One example of an LCLS experiment is to use an x-ray laser, which will hit a target material and detectors will take pictures of the resulting diffraction pattern. Short bunches and precise timing at the femtosecond level will be used to take digital images [4]. Images from different beam pulses with femtosecond timing information will then be put together to show 
how the reaction occurred in real time (3).

The tools to accomplish this task will be the Matlab and the Litrack programs. Litrack was specifically designed to study the electron beam's longitudinal emittance and how it evolves during beam transport through SLAC’s linac [5]. Litrack program can adjust parameters (such as phase of the beam with respect to the accelerating rf in different sections of the Linac) of different beamlines to study the dependence of the bunch length distribution on these (2) LiTrack is run from Matlab, which is an analysis package using linear algebra and matrix manipulation for modeling and creating output files and plots (1).

\section{MATERIALS AND METHODS}

For these simulations, we used the Matlab and the LiTrack programs to simulate the ILCESA beamline, LCLS beamline, LCLS-ESA beamline and there parameters.

To determine the sensitivity of the rms bunch length, we looked at the effects caused by changes in certain parameters. The first parameter changed was the phase degree of the acceleration phase, which determines where the electron bunch is located with respect to the peak of the rf voltage wavelength (Figure 3). The phases degree was adjusted for the acceleration phase of Sector 2 thru Sector 10 for the ILC-ESA beamline and Linac-A for the LCLS and LCLS-ESA beamlines. The next parameter to be varied was the phase degree of the acceleration phase of Sectors 10-30 for the ILC beamline and Linac-B for the LCLS and LCLS-ESA beamlines. The last parameter to be studied is the compressor voltage. The compressor voltage is the voltage that an electron bunch has when the bunch reaches the peak of the rf voltage (see Figure 3).

Table 1 show the basic command and code for the beamline. The first column displays 
the specific action that will be carried on. These names are not included in beamline matrices. The second column shows the specific number code for the action. The other five columns describe distinct characteristics of the particular code at that given moment. The 10 and 11 code show how where the beam is located (last value in the row). This is significant for changing parameter in given sections.

Using the LiTrack program, we changed the acceleration phase for ILC-ESA beamline file in Sector 2 thru Sector 10 of the Linac from the default value of $10^{\circ}$ to values $+-1^{\circ},+-2^{\circ}$, and $+-3^{\circ}$ of the default value. The default value was found by looking at Table 2 for a phase value in Sector 2. The change in phase degree value illustrates how the rms bunch length, FIT ( a Gaussian fit that compare the Zpos and current in the form of a bell curve), and peak current are affected. The data was collected form the figure which shows the result that would be produced in ESA. From the information gathered, we noticed the fit value had a negative slope. This information caused for the inquiry of the further information about the phase at it increase by increments greater than $3^{\circ}$. Information was gathered for the ILC-ESA until slope of the fit became positive. The information was then recorded; we continued collecting data for the ILCESA for Sector 10 thru Sector 30 of the Linac. In these sectors, we changed the default phase $17.5^{\circ}$ to values $+-1^{\circ},+-2^{\circ}$, and $+-3^{\circ}$ of the default values. The default value was found by looking at Table \# for a phase value in Sector 11. The results can be seen in figure 5 and 6.

We now look at the effects that the compressor voltage has on the FIT, rms bunch length and peak current in the ILC-ESA. The phase degree in the acceleration phase was left at $10^{\circ}$ in Sector 2 thru 10 and at $16.5^{\circ}$ degrees in Sectors 10 thru 30 of Linac. The compressor voltage was change from $+-1 \mathrm{MeV},+-2 \mathrm{MeV}$, and $+-3 \mathrm{MeV}$ of the default value, which is $38.5 \mathrm{MeV}$. The default value was found by looking at Table 2 for a phase value in Sector 2 . The data was 
collected from the figure, which displayed the results that are produced in ESA. The result are seen in Figure 11.

After testing the ILC, we now test the sensitivity of the LCLS. Using Table 3 we found the acceleration phase for Linac-A of LCLS. We then changed the phase degree of the LCLS $+-1^{\circ},+-2^{\circ}$, and $+-3^{\circ}$ of the default value of $40.8^{\circ}$. The results used are the ones that correspond with the end of the LCLS and can be seen in Figure 7. Next, we repeated the procedure for the LCLS, except changing the default value $\left(13.58^{\circ}\right)$ of Linac-B by $+-1^{\circ},+-2^{\circ}$, and $+-3^{\circ}$. The default value was found in Table 3. The results used are the ones that correspond with the end of the LCLS and can be seen in Figure 8.

The last beamliine tested was the LCLS-ESA. The LCLS-ESA test methods are similar to the LCLS beamline, but a few differences. The default values came from Table 4 and are $10.8^{\circ}$ for the Linac-A and $11^{\circ}$ for Linac-B. The result correspond to the result produce in ESA and found in Figures 9 and 10.

Next we look at the longitudinal position (Zpos) and the relative energy ( ) of the bunches generated from LiTrack. This part of simulation we involved some computer programming use $\mathrm{C}++$ in Matlab. In order to complete the task we must first add a code 2 (code 2 is a code that dumps Zpos and relative energy into an ASCII file) to the beamline parameter file [5]. Once this is complete, run Litrack and a new file called "litrack_zd_output.dat” is generated. The next step was to create a program called “Zpos\&E” that would use the litrack_zd_output file to calculate the standard deviation (std), mean, a Zpos histogram, a histogram, and a plot showing zpos verse . Once the program was completed, we began working on creating a program called “LiTrack-loop” that will repeat LiTrack a finite number of 
times. The next step was to make a program called “G-Fit” that would create a Gaussian Fit for the zpos and place the Fit over a plot of the current vs. the Zpos. The final step was to add the 3 small programs created and make one program that will complete all these task.

\section{RESULTS}

Figure 5 shows the rms bunch length and peak current for the ILC in Sectors 2-10. The peak current $\left(\mathrm{I}_{\mathrm{pk}}\right)$ increases until the phase degree reaches $18.5^{\circ}$ at the point the peak reaches a high of $1.42 \mathrm{kA}$. The fit (fit rms) starts with a value of $0.468 \mathrm{~mm}$ at $8^{\circ}$ and continues to decrease until the phase degree reaches $19.5^{\circ}$ at this particular point the fit is a value of $0.128 \mathrm{~mm}$. In

Figure 4 , we see that the phase degree has to decrease by $5^{\circ}$ before the bunch length decrease by a little more than half. This rate continues until the bunch length reaches its relative minimum value. Once the relative minimum is achieved, the bunch length began increasing at the same rate.

Figure 6 shows the rms bunch length and peak current for the ILC in Sectors 10-30. The $\mathrm{I}_{\mathrm{pk}}$ show an increasing current until the current reaches a value of $1.4403 \mathrm{kA}$. The current occurs when the phase is at $19.5^{\circ}$. The rms fit begins at $15.5^{\circ}$ with a magnitude of $0.497 \mathrm{~mm}$. At $19.5^{\circ}$, the rms fit stops decreasing and reaches a relative minimum of $0.132 \mathrm{~mm}$. Figure 5 shows the phase degree Sectors 10 - 30, which decrease by half every two degrees. As with Sector 2- 10, this continues until it reaches the minimum. The $\mathrm{I}_{\mathrm{pk}}$ for both sections is at a relative max around the same time the bunch length reaches their minimal values.

Figure 11 displays the results for the ILC when the compressor voltage is varied. The rms fit goes up and down from1.933 mm to $0.212 \mathrm{~mm}$, until $41.5 \mathrm{MeV}$. At $41.5 \mathrm{MeV}$, the rms fit shoots up to $22017 \mathrm{~mm}$. The $\mathrm{I}_{\mathrm{pk}}$ shows a curve similar to trig function with a maximum of 0.9615 
$\mathrm{kA}$ at $38.5 \mathrm{kA}$. The minimum values occur at $35.5 \mathrm{MeV}$ and $40.5 \mathrm{MeV}$ with a corresponding voltage of $0.1762 \mathrm{kA}$ and $0.1870 \mathrm{kA}$. Figure 10 shows the affects that a change in compressor voltage would have on the ILC. The figure shows a very unstable rms bunch length, which seems to increase and then decease at a random length each change. The $I_{p k}$ increase about $1 \mathrm{kA}$ every three degrees and then follow by decreasing at the same rate.

The LCLS, shown in Figure 7, starts with a rms bunch length of $40.821 \mathrm{~m}$ and an $\mathrm{I}_{\mathrm{pk}}$ of $4.318 \mathrm{kA}$ at a phase degree of $38.8^{\circ}$. The rms's relative minimum is $3.549 \mathrm{~m}$, which occurs at $41.8^{\circ}$. At the same point, the $\mathrm{I}_{\mathrm{pk}}$ reaches it relative maximum of $36.728 \mathrm{kA}$. The LCLS results for Linac-A show that the rms bunch length decreased to one-tenth of the bunch length at $38.8^{\circ}$ in a three degree change (see Figure 7). The $\mathrm{I}_{\mathrm{pk}}$ experience the same change in values except the change was an increase in kA. Figure 8 explains the results for Linac-B. In this figure, we see a change of $0.4 \mathrm{~m}$ in $6^{\circ}$. The peak current a change of $-4 \mathrm{kA}$ occur in the same duration.

Figure 9 shows the LCLS-ESA beamline for Linac-A. In this figure, the rms and rms fit seems to be a constant function set at $183 \mathrm{~m}$ and $0.018 \mathrm{~mm}$. The $\mathrm{I}_{\mathrm{pk}}$ oscillates between $3.3 \mathrm{kA}$ and 3.5 kA during the different phases. In Figure 9 we see the results of the LCLS-ESA for Linac A. The figure shows that rms bunch length is not affected by a change in degree phase. The peak current shows similar result to the rms bunch length.

In Figure 10, the rms fit starts at $\mathrm{mm}$ and the $\mathrm{I}_{\mathrm{pk}}$ starts at 2.142kA for the LCLS-ESA in Linac-B. The rms fit reaches a relative minimum of $0.018 \mathrm{~mm}$ when the phase is $11^{\circ}$. At $11^{\circ}$, the $\mathrm{I}_{\mathrm{pk}}$ reaches a relative maximum of $3.506 \mathrm{kA}$. The rms bunch length in Linac-B shows the length decreasing to one-forth of the value at $9^{\circ}$ in two degrees (see Figure 10). Yet, the length increase at an even larger rate. The current continues the trend of acting opposite of the rms bunch length. 


\section{DISCUSSION AND CONCLUSION}

The results show the bunch length being affected most when the acceleration phase for Linac-A of the LCLS were changed. This was determined by looking at the ratio for the change in phase degree to rate of change in bunch length. The ratio for the LCLS in Linac-A was an astonishing 3:10. The LCLS-ESA in Linac-B was the next in sensitivity having a ratio of 2:4. The rest in order of sensitivity were the ILC in Sectors $10-30$, ILC in Sectors $2-10$, LCLS in Linac-B, and LCLS-ESA in Linac-A (see Table 5). The LCLS-ESA in Linac-A was the least sensitive having a ratio of $6: 0^{+}$. The compressor voltage showed very rapid changes in bunch length; however, the rate of change was so sporadic that I was unable to clarify a clear rate of change. The peak current for the change in phase degree showed the same rate of changes as the bunch length. The peak current for the compressor voltage show a ratio of change equivalent to 3:5.

The results from Figure 5-10, show that peak current was inversely proportional to the fit rms value. This property suggests that the current might be able to detect when the bunch will have the lowest length. If this property holds true, then the absolute minimum in rms bunch length can be calculated by observing the peak current as the phase degree change. The highest peak current should be around the area of the lowest bunch length.

The LCLS beamline and LCLS-ESA beamline are identical lines, except for the LCLSESA continuing down to ESA and different compressor voltage. The compressor voltage for the LCLS and LCLS-ESA only varies in the Linac-A section. In this section, the voltage is $4.5 \mathrm{GeV}$ in LCLS and 5.7 GeV in the LCLS-ESA. This gives a small explanation for the different results, but does not explain the result thoroughly because the changes caused by the ESA are not shown. 
Suggesting further looks at the affects of the A-line and ESA

In this project, we have studied the sensitivity of bunch length distribution for three planned beamlines at SLAC to different acceleration phase and compressor voltage.

\section{ACKNOWLEDGEMENT}

This research was conducted at the Stanford Linear Accelerator Center. I would like to thank the Department of Energy, Office of Science for allowing me to participate in the SULI program and giving me an exciting and knowledgeable experience. I would also like to Helen Quinn, James Lindesay, and the SULI participants for 2005, who got me involved in this programmed and gave me words of wisdom when they were needed. Special thanks go to my mentor Michael Woods, who share his knowledge in 2-D simulation, computer programming, and Linear Accelerators.

\section{REFERENCES}

[1] M. Woods, R. Erickson, J. Frisch, "A test facility for the ILC at SLAC ESA, for prototypes of beam Delivery and IR components," for Particle Accelerator Conference (PAC), Knoxville, TN, May, 2005

[2] P. Emma, M. Woods, "Electron bunch length compression in the SLAC A-line," IPBI TN-2004-8, August, 2004

[3] P. Krejcik, "Short bunch beam diagnostics,” SLAC-PUB-9527, September, 2002

[4] J. Galayda, H.R Woods, "Linac Coherent Light Source: New tools create new science,” April, 2005

[5] K.L.F. Banes, P. Emma, "LiTrack: A fast longitudinal phase space tracking code with graphical user interface,” for PAC, Knoxville, TN, May, 2005 


\section{TABLES}

\begin{tabular}{|l|c|c|c|c|c|c|}
\hline descrip. & code & $p_{1}$ & $p_{2}$ & $p_{3}$ & $p_{4}$ & $p_{5}$ \\
\hline mark & 1 & & & & & \\
dump & 2 & & & & & \\
compr. & 6 & $R_{56}$ & $T_{566}$ & $E_{0}$ & $U_{5666}$ & \\
chicane & 7 & $R_{56}$ & $E_{0}$ & & & \\
linac & 10 & $E_{\text {final }}$ & $\phi$ & $\lambda$ & $i_{\text {wake }}$ & $L$ \\
linac & 11 & $V_{0}$ & $\phi$ & $\lambda$ & $i_{\text {wake }}$ & $L$ \\
rw-wake & 15 & $\sigma$ & $L$ & $\sigma_{c}$ & $\tau$ & $1 / 0$ \\
ISR & 22 & $\sigma_{\delta}$ & & & & \\
E-cuts & 26 & $\delta_{\min }$ & $\delta_{\max }$ & & & \\
$z$-cuts & 36 & $z_{\min }$ & $z_{\max }$ & & & \\
STOP & 99 & & & & & \\
\hline
\end{tabular}

Table 1. List of function-codes and their parameters for LiTrack. All units are marked except energy, voltage, and phase, which are GeV, GV, and degrees, respectively; $\pm^{\prime} \$ E=E 0$. Blank parameter slots are not used by the code.

\begin{tabular}{|l|l|l|l|l|l|}
\hline-11 & 0 & 0 & 0.104969 & 0 & 0 \\
\hline-11 & 0.385 & 90.0 & 0.104969 & 1 & 2.13 \\
\hline 26 & -0.021 & 0.021 & 0 & 0 & 0 \\
\hline-6 & 0.590 & 1.0535 & 1.190 & 0 & 0 \\
\hline$-10^{*}$ & 9.000 & -10.5 & 0.104969 & 1 & 809.5 \\
\hline$-10^{\wedge}$ & 28.500 & -17.5 & 0.104969 & 1 & 1872.4 \\
\hline-6 & 0.465 & 2.744 & 28.500 & 0 & 0 \\
\hline-22 & $1.0 \mathrm{E}-4$ & 0 & 0 & 0 & 0 \\
\hline-26 & -0.005 & 0.005 & 5 & 0 & 0 \\
\hline 2 & 0 & 0 & 0 & 0 & 0 \\
\hline-99 & 0 & 0 & 0 & 0 & 0 \\
\hline
\end{tabular}

Table 2. Shows the beamline code use to simulate the ILC in LiTrack program. * = Sector 2$10, \wedge=$ Sector $10-30$ 


\begin{tabular}{|l|l|l|l|l|l|}
\hline-11 & 0 & 0 & 0.104969 & 0 & 0.1 \\
\hline-11 & 0.12959 & -1.1 & 0.104969 & 1 & 6.1 \\
\hline 22 & $3.50 \mathrm{E}-4$ & 0 & 0 & 0 & 0 \\
\hline-6 & 0.0063 & 0.140 & 0.135000 & 0 & 0 \\
\hline-11 & 0.14739 & -20 & 0.104969 & 1 & 8.78 \\
\hline-11 & 0.0190 & -160 & 0.026242 & 2 & 0.60 \\
\hline-7 & -0.03905 & .25 & 0 & 0 & 0 \\
\hline$-11^{*}$ & 5.71031 & 40.8 & 0.104969 & 1 & 329.1 \\
\hline 7 & -0.0247 & 4.54 & 0 & 0 & 0 \\
\hline-22 & $0.80 \mathrm{E}-5$ & 0 & 0 & 0 & 0 \\
\hline $11^{\wedge}$ & 9.9145 & 13.58 & 0.104969 & 1 & 552.9 \\
\hline 11 & 0 & 0 & 0.105 & 5 & 76 \\
\hline 11 & 0 & 0 & 0.105 & 6 & 275 \\
\hline 6 & 0.000133 & 0.0063 & 14.1 & 0 & 0 \\
\hline-22 & $1.32 \mathrm{E}-5$ & 0 & 0 & 0 & 0 \\
\hline 27 & 0.020 & 1 & 0 & 0 & 0 \\
\hline-99 & 0 & 0 & 0 & 0 & 0 \\
\hline
\end{tabular}

Table 3. Shows the beamline code use to simulate the LCLS in LiTrack program. * = Linac-A, $\wedge=$ Linac-B 


\begin{tabular}{|l|l|l|l|l|l|}
\hline-11 & 0 & 0 & 0.104969 & 0 & 0.1 \\
\hline-11 & 0.12959 & -1.1 & 0.104969 & 1 & 6.1 \\
\hline 22 & $3.50 \mathrm{E}-4$ & 0 & 0 & 0 & 0 \\
\hline-6 & 0.0063 & 0.140 & 0.135000 & 0 & 0 \\
\hline-10 & 0.26915 & -25 & 0.104969 & 1 & 8.78 \\
\hline-11 & 0.0190 & 160.0 & 0.026242 & 2 & 0.6 \\
\hline-7 & 0.03905 & 0.2500 & 0 & 0 & 0 \\
\hline$-10^{*}$ & 4.574 & 10.8 & 0.104969 & 1 & 329.1 \\
\hline 7 & -0.0247 & 4.54 & 0 & 0 & 0 \\
\hline-22 & $0.80 \mathrm{E}-5$ & 0 & 0 & 0 & 0 \\
\hline$-10^{\wedge}$ & 14.21 & 11 & 0.104969 & 1 & 552.9 \\
\hline 6 & 0 & 0.229 & 14.1 & 0 & 0 \\
\hline 22 & 0 & 0 & 0 & 0 & 0 \\
\hline 6 & 0.03875 & 0.229 & 14.1 & 0 & 0 \\
\hline 22 & $1.01 \mathrm{E}-5$ & 0 & 0 & 0 & 0 \\
\hline 6 & 0.03875 & 0.229 & 14.1 & 0 & 0 \\
\hline 22 & $1.01 \mathrm{E}-5$ & 0 & 0 & 0 & 0 \\
\hline 6 & 0.03875 & 0.229 & 14.1 & 0 & 0 \\
\hline 22 & $1.01 \mathrm{E}-5$ & 0 & 0 & 0 & 0 \\
\hline 6 & 0.03875 & 0.229 & 14.1 & 0 & 0 \\
\hline 22 & $1.01 \mathrm{E}-5$ & 0 & 0 & 0 & 0 \\
\hline 6 & 0.03875 & 0.229 & 14.1 & 0 & 0 \\
\hline 22 & $1.01 \mathrm{E}-5$ & 0 & 0 & 0 & 0 \\
\hline 6 & 0.03875 & 0.229 & 14.1 & 0 & 0 \\
\hline 22 & $1.01 \mathrm{E}-5$ & 0 & 0 & 0 & 0 \\
\hline 6 & 0.03875 & 0.229 & 14.1 & 0 & 0 \\
\hline 22 & $1.01 \mathrm{E}-5$ & 0 & 0 & 0 & 0 \\
\hline 6 & 0.03875 & 0.229 & 14.1 & 0 & 0 \\
\hline 22 & $1.01 \mathrm{E}-5$ & 0 & 0 & 0 & 0 \\
\hline 6 & 0.03875 & 0.229 & 14.1 & 0 & 0 \\
\hline 22 & $1.01 \mathrm{E}-5$ & 0 & 0 & 0 & 0 \\
\hline 6 & 0.03875 & 0.229 & 14.1 & 0 & 0 \\
\hline 22 & $1.01 \mathrm{E}-5$ & 0 & 0 & 0 & 0 \\
\hline 6 & 0.03875 & 0.229 & 14.1 & 0 & 0 \\
\hline 22 & $1.01 \mathrm{E}-5$ & 0 & 0 & 0 & 0 \\
\hline 6 & 0.03875 & 0.229 & 14.1 & 0 & 0 \\
\hline-22 & $1.01 \mathrm{E}-5$ & 0 & 0 & 0 & 0 \\
\hline 36 & $1.9 \mathrm{e}-3$ & $2.3 \mathrm{e}-3$ & 0 & 0 & 0 \\
\hline-99 & 0 & 0 & 0 & 0 & 0 \\
\hline & & & 0 & 0 \\
\hline
\end{tabular}

Table 4. Shows the beamline code use to simulate the LCLS-ESA in LiTrack program. * $=$ Linac-A, $\wedge=$ Linac-B 


\begin{tabular}{|l|l|l|}
\hline \multicolumn{1}{|c|}{ Beamline } & \multicolumn{1}{|c|}{$\begin{array}{c}\text { Minimum Bunch Length } \\
(\mathrm{m})\end{array}$} & \multicolumn{1}{c|}{$\begin{array}{c}\text { Sensitivity Ratio } \\
\text { (degrees : rate of change) }\end{array}$} \\
\hline ILC Sectors 2-10 & 128 & $5: 2$ \\
\hline ILC Sectors 10-30 & 132 & $2: 2$ \\
\hline LCLS Linac-A & 3.549 & $3: 10$ \\
\hline LCLS Linac-B & 16.438 & $6: 0.9$ \\
\hline LCLS-ESA Linac-A & 15 & $6: 0$ \\
\hline LCLS-ESA Linac-B & 18 & $2: 4$ \\
\hline ILC voltage & 212 & NA \\
\hline
\end{tabular}

Table 5. Show the sensitivity ratio for each beam line and the lowest bunch length produced by each beamline.

\section{FIGURES}

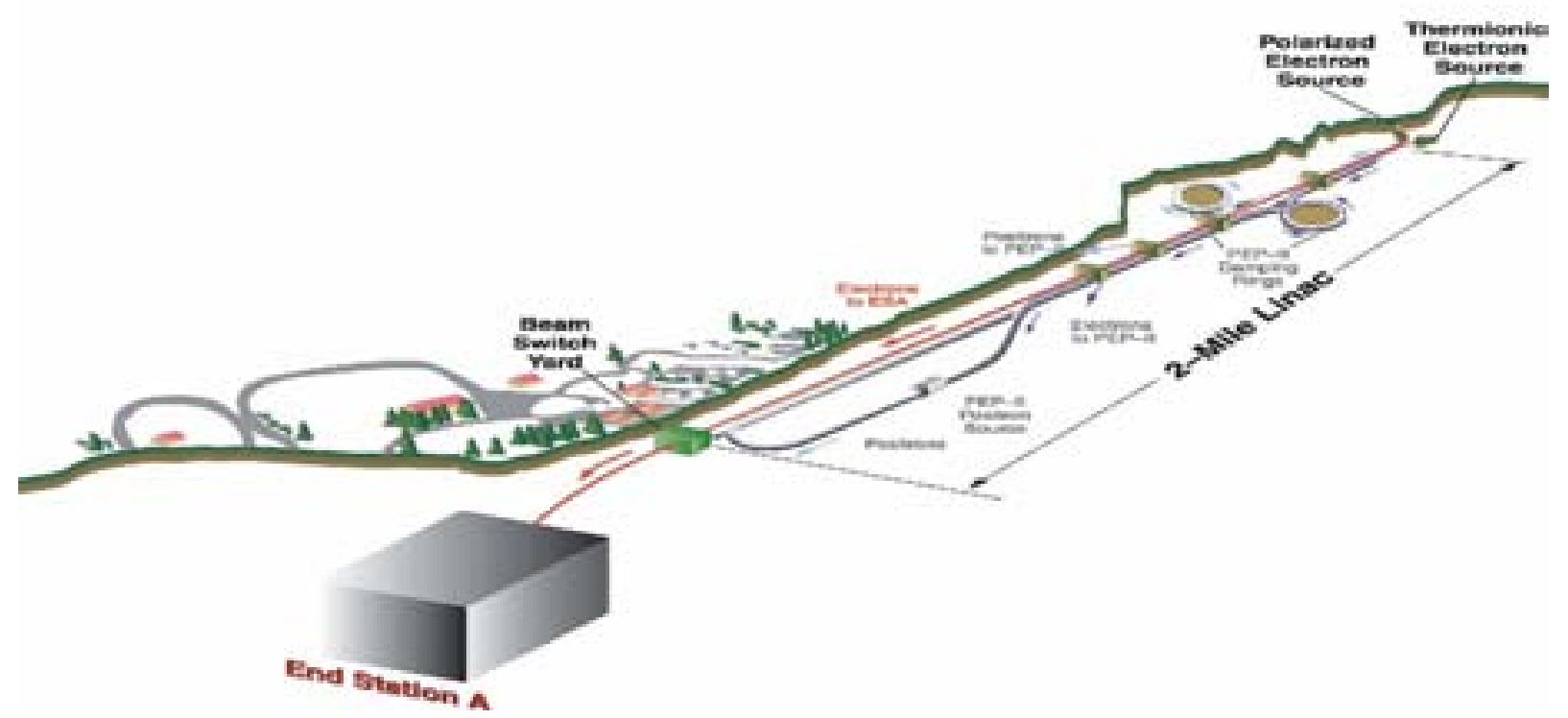

Figure 1. The Set up of the Linear Accelerator at SLAC. 


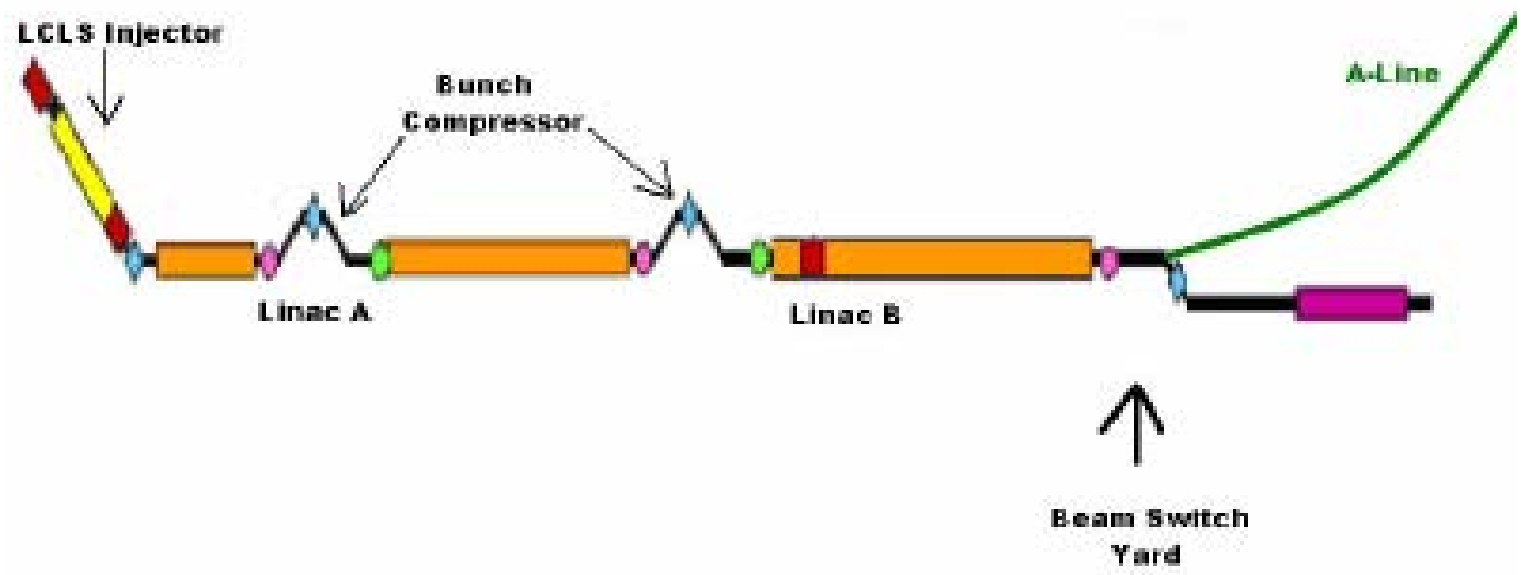

\section{LCLS Beamline}

Figure 2.

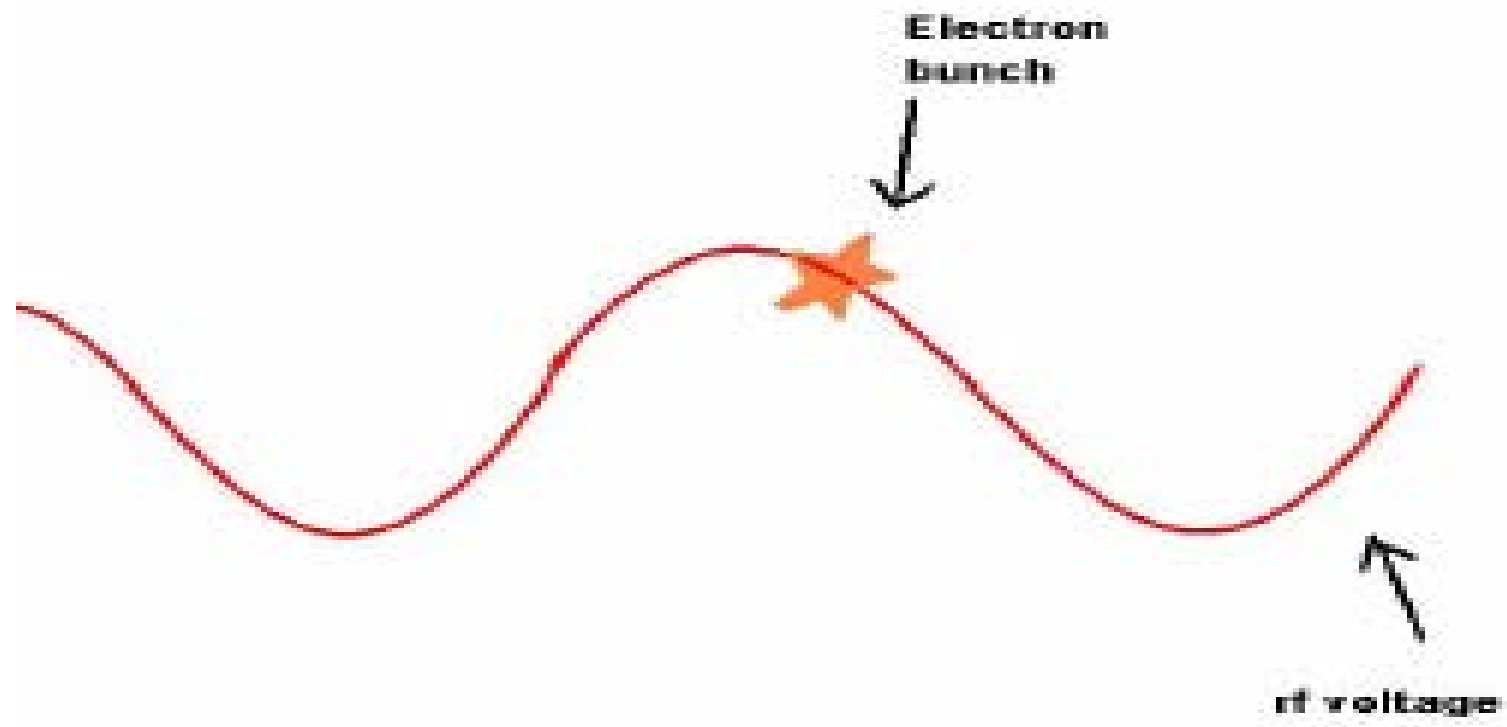

Figure 3. 

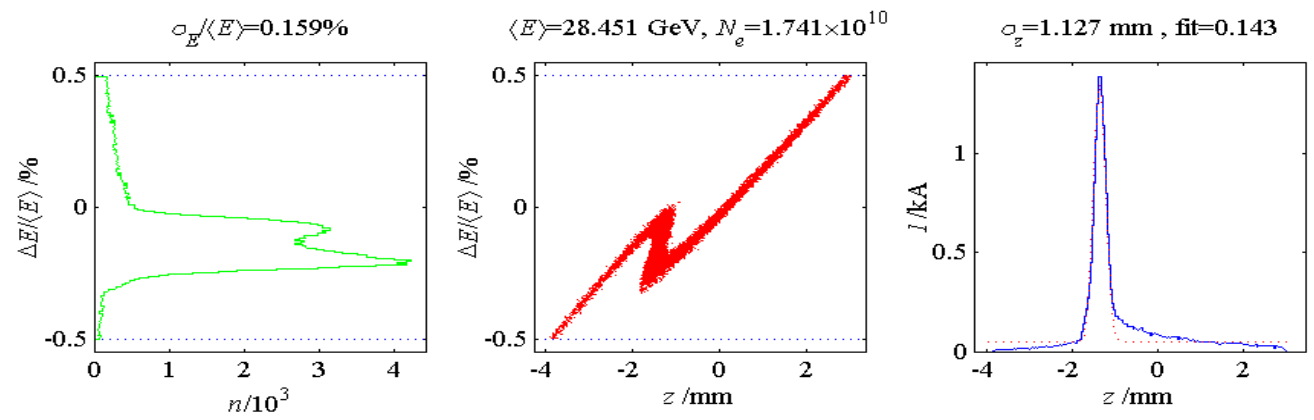

Aline to ILC Sector 217 Sector $_{10} 16$

Figure 4. Example of the results produced LiTrack program.. The left figure shows a histogram of $\mathrm{E} / \mathrm{E}$. The center figure shows a plot of $\mathrm{E} / \mathrm{E}$ vs. Zpos. The figure on the right shows a plot of Zpos vs. current. The fit and rms fit are located above the figure on the far right.

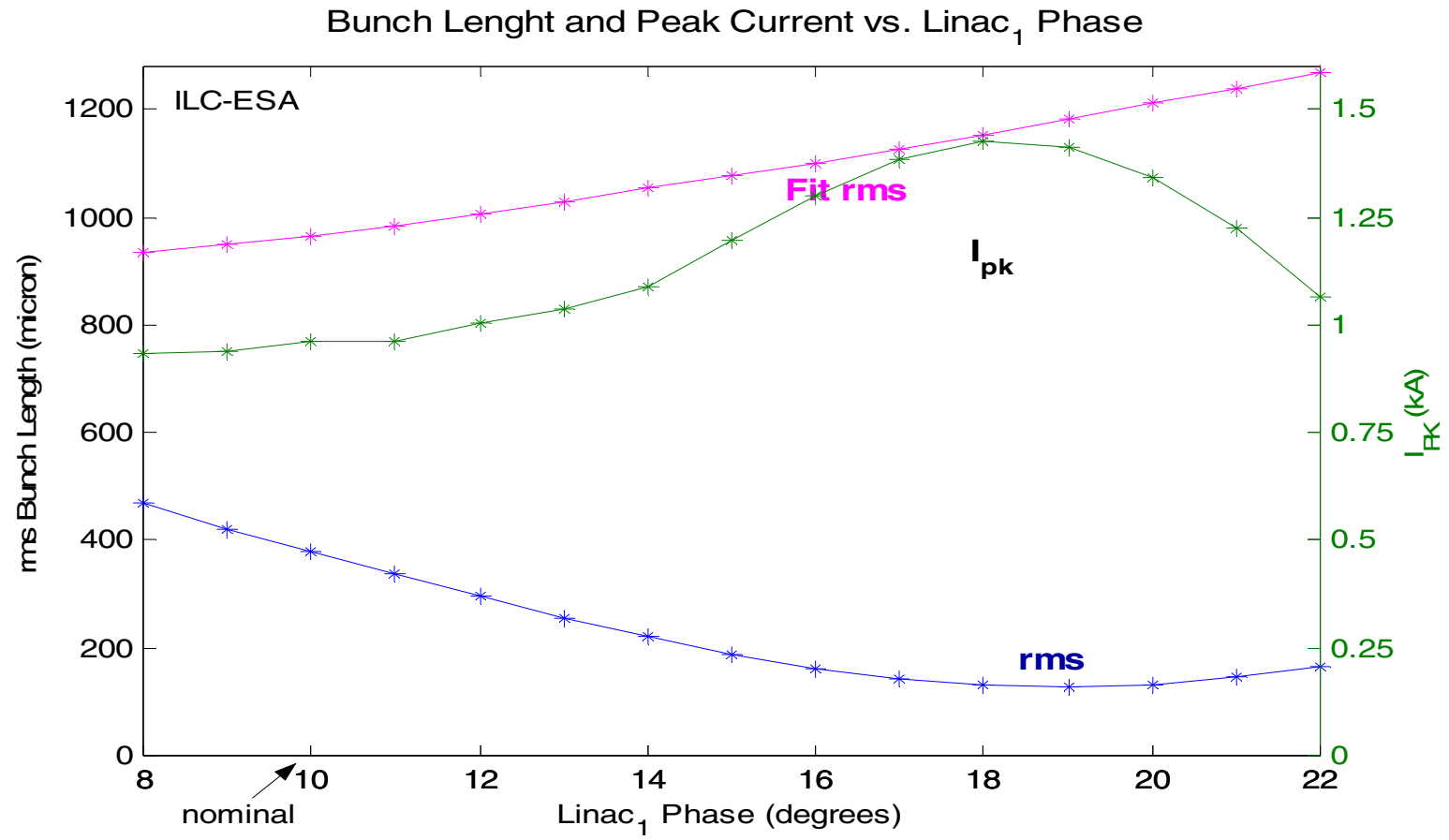

Figure 5. Compares the rms bunch length, fit, and peak current as the phase changes Sector 2 thru 10 of the ILC-ESA. In Sectors $10-30$ the phase is $16.5^{\circ}$ and the compressor voltage is 38.5 $\mathrm{MeV}$ 


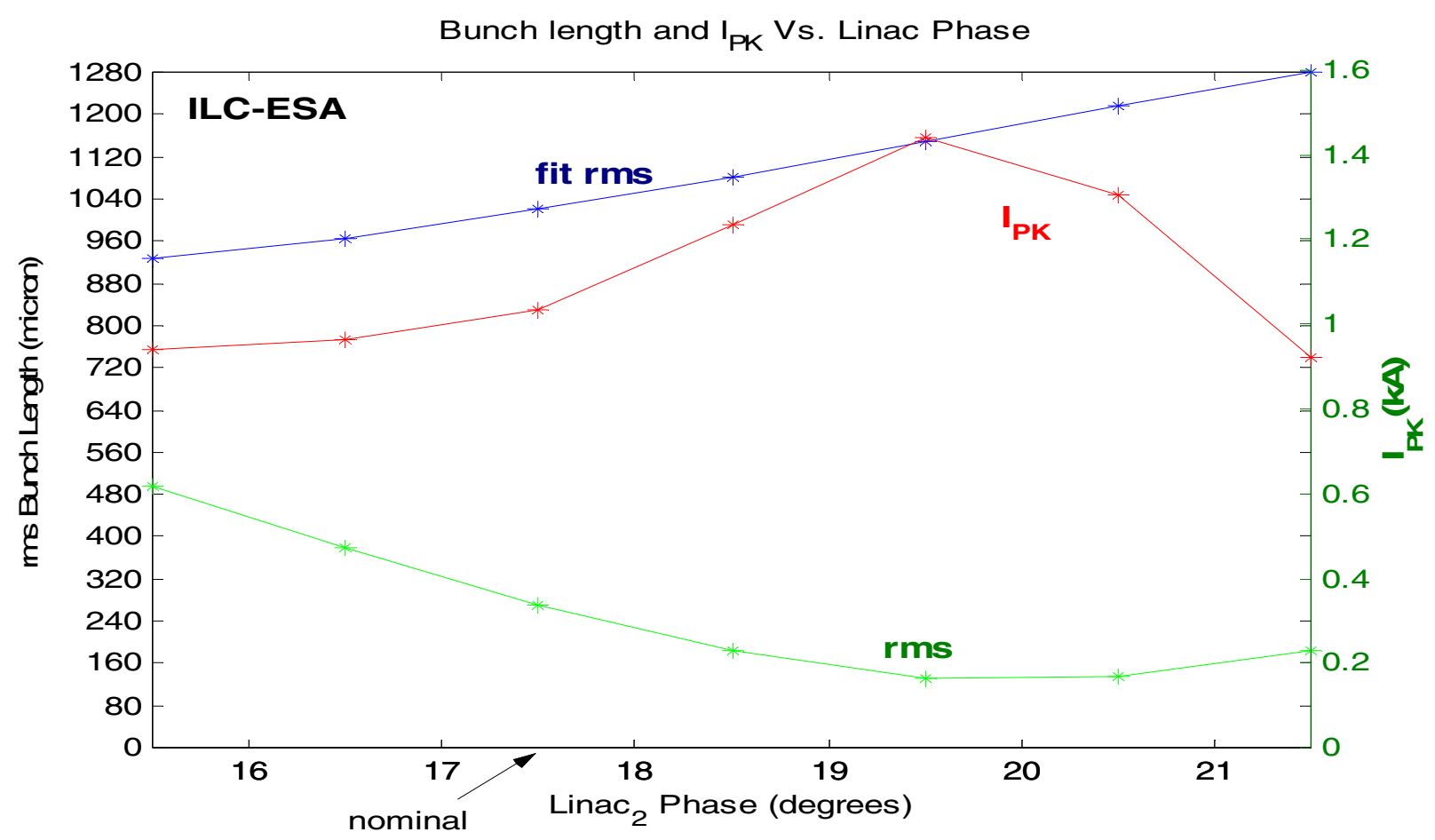

Figure 6. Compares the rms bunch length, fit, and peak current as the phase changes Sector 10 thru 30 of the ILC-ESA. . In Sectors $2-10$ the phase is $10^{\circ}$ and the compressor voltage is 38.5 $\mathrm{MeV}$

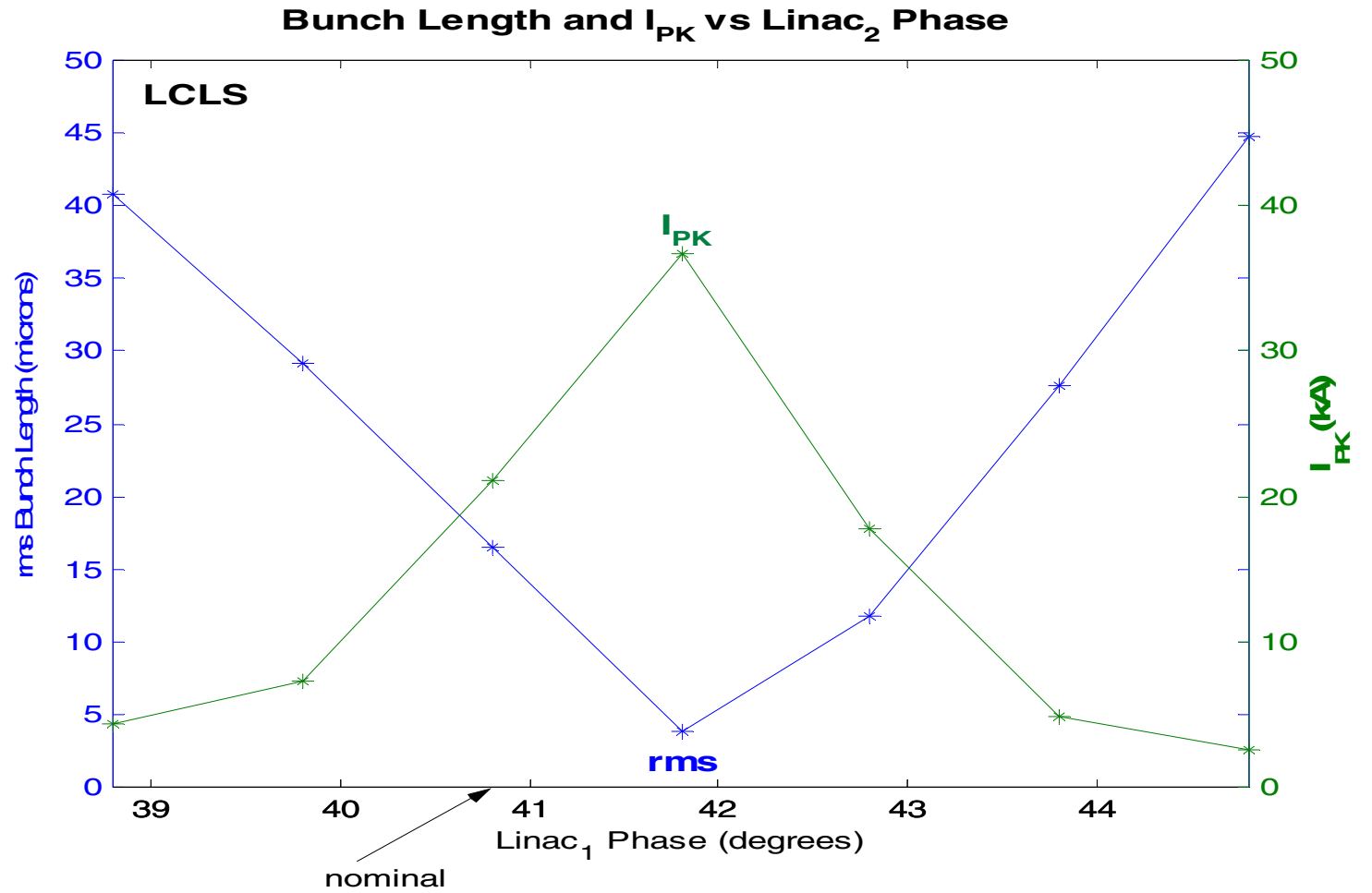

Figure 7. Compares the rms bunch length, fit, and peak current as the phase changes in Linac-A of the LCLS. In Linac-B the phase is $13.58^{\circ}$ and the compressor voltage is $4.5 \mathrm{GeV}$ 


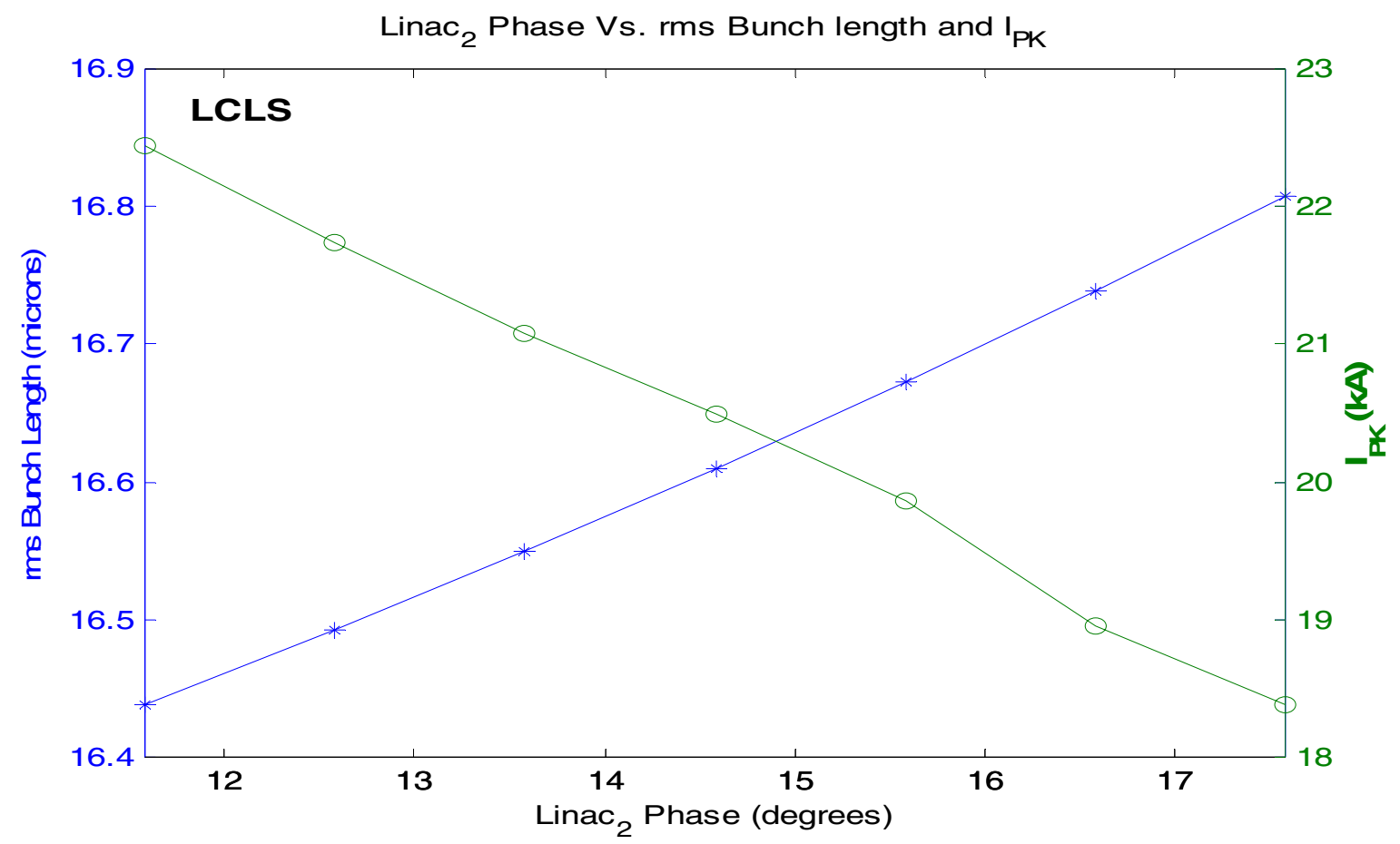

Figure 8. Compares the rms bunch length, fit, and peak current as the phase changes in Linac-B of the LCLS. . In Linac-A the phase is $40.8^{\circ}$ and the compressor voltage is $9.5 \mathrm{GeV}$

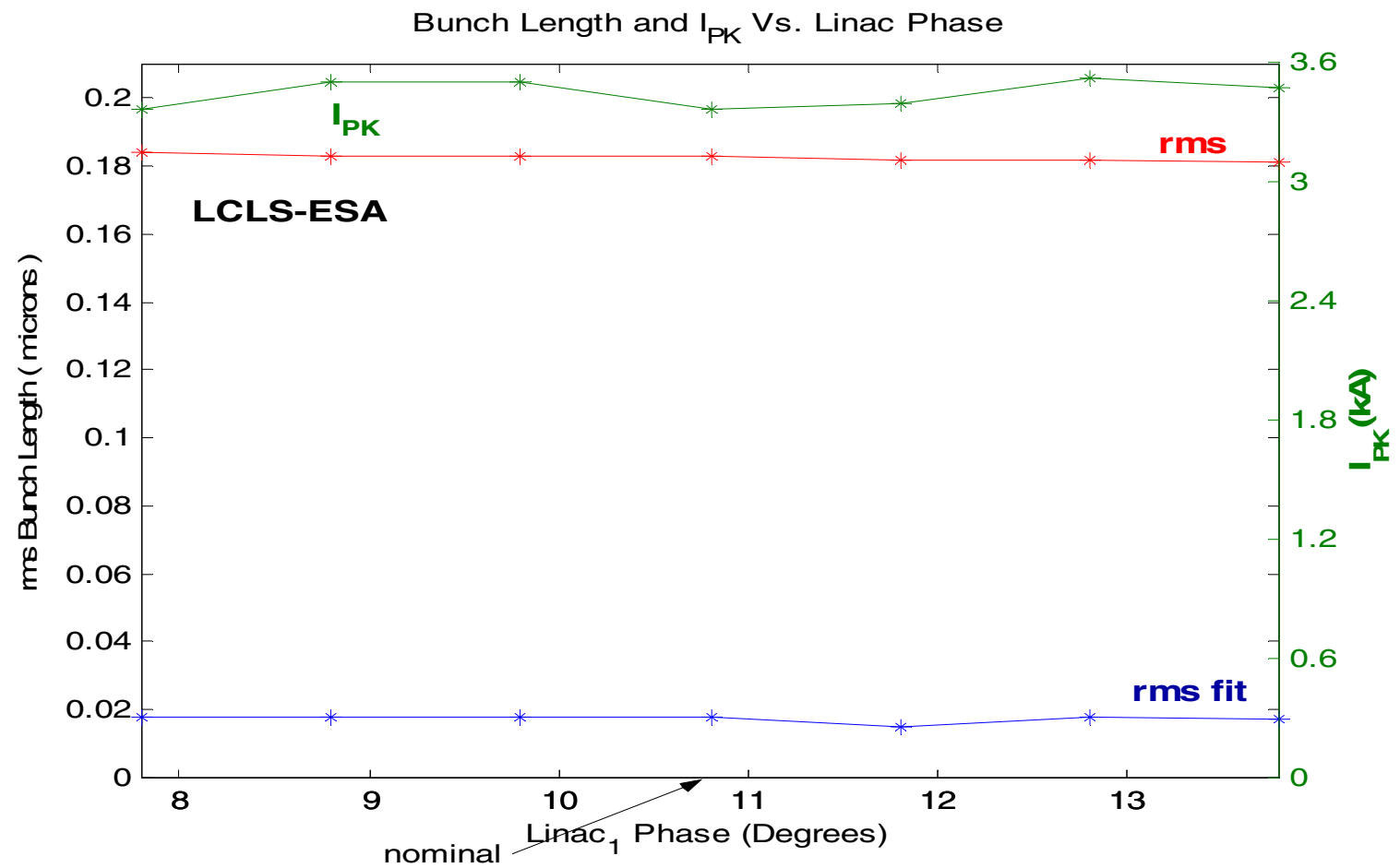

Figure 9. Compares the rms bunch length, fit, and peak current as the phase changes in Linac-A of the LCLS-ESA. In Linac-B the phase is $10^{\circ}$ and the compressor voltage is $9.9 \mathrm{GeV}$ 
Bunch Length and $\mathrm{I}_{\mathrm{PK}}$ Vs. Linac Phase

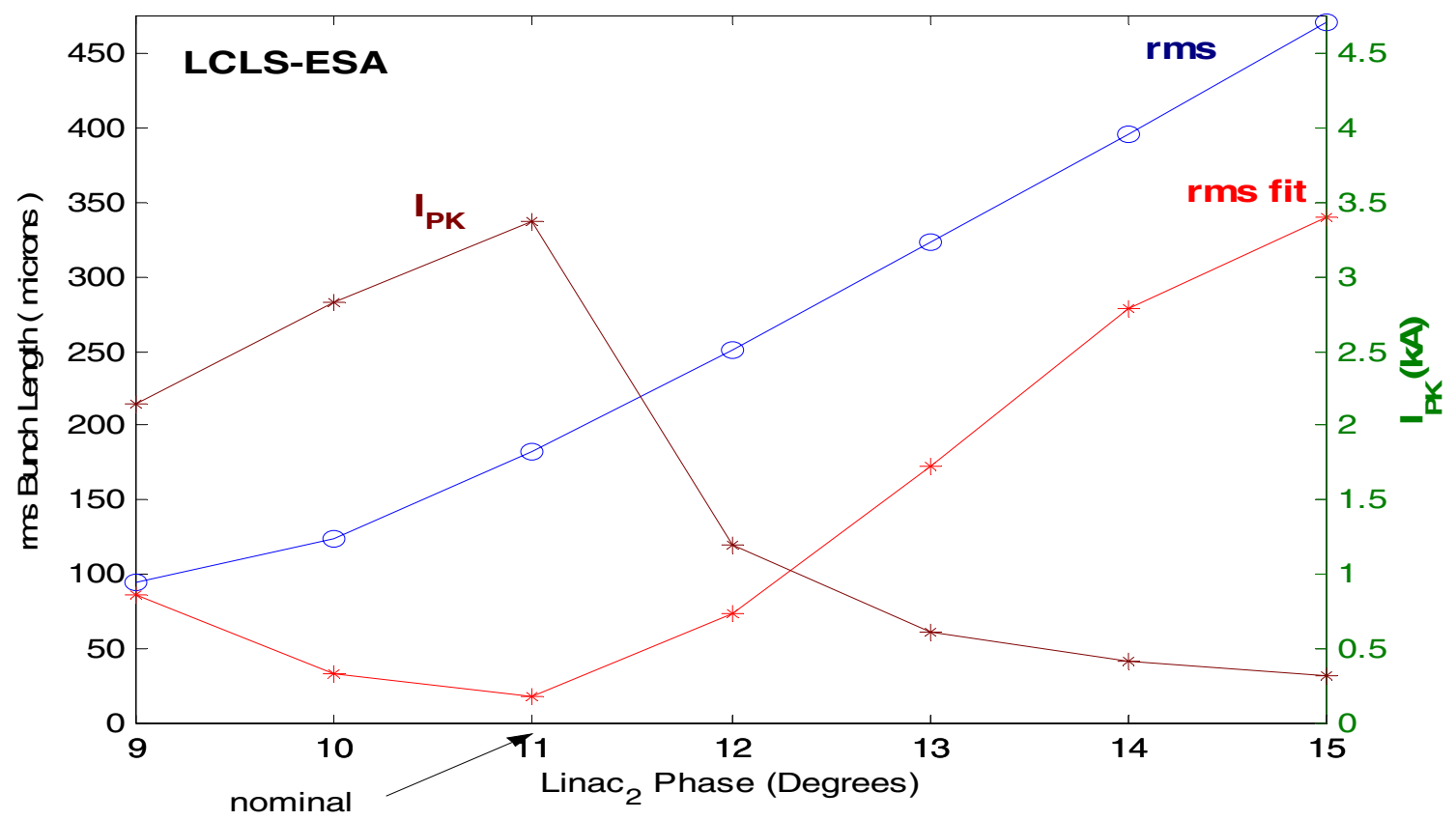

Figure 10. Compares the rms bunch length, fit, and peak current as the phase changes in Linac-B of the LCLS-ESA. . In Linac-A the phase is $10.8^{\circ}$ and the compressor voltage is $14.1 \mathrm{GeV}$

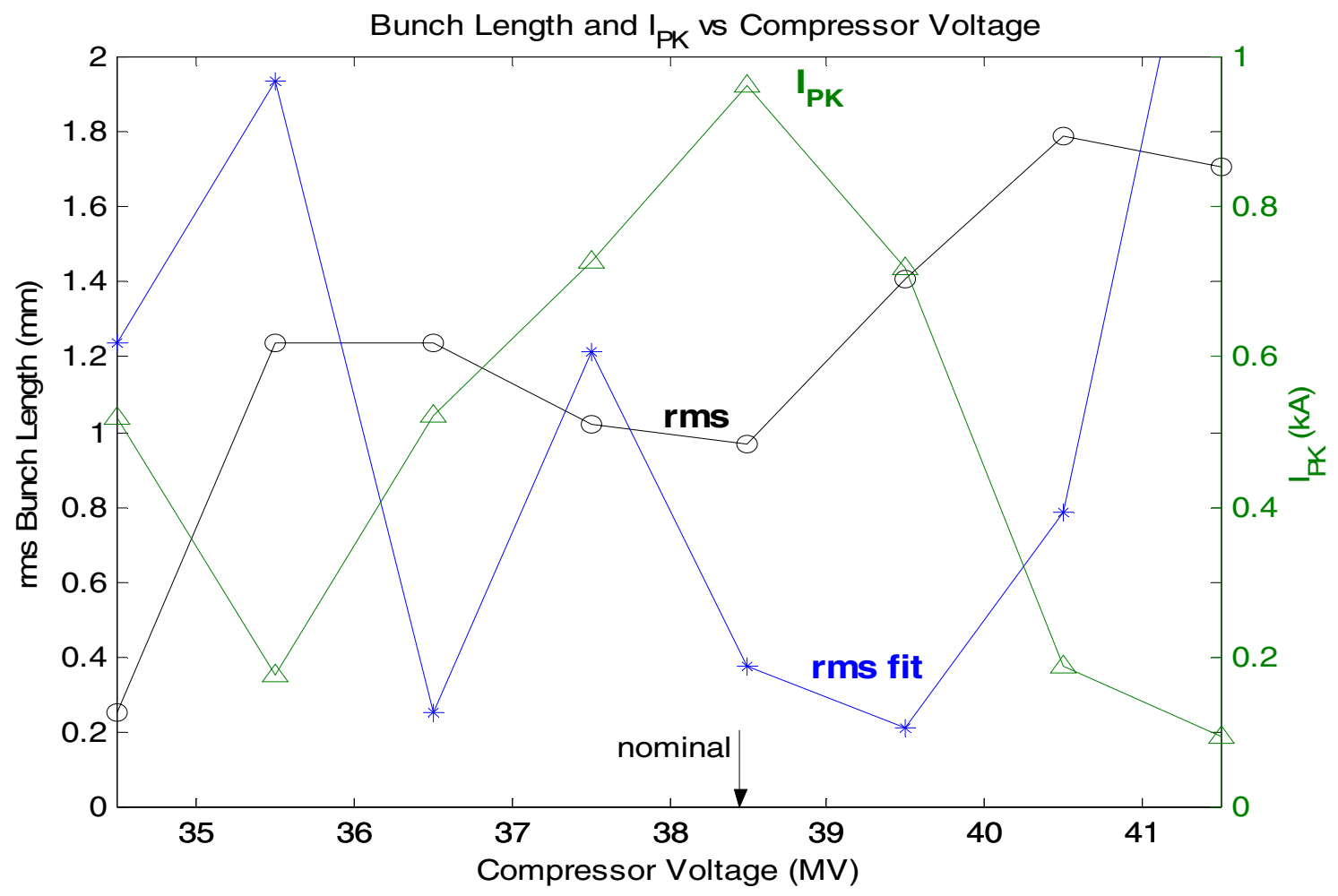

Figure 11. Compares the rms bunch length, fit, and peak current as the compressor voltage of the ILC-ESA changes. Sectors $2-10$ phase is $10.8^{\circ}$ and Sectors $10-30$ phase is $16.5^{\circ}$. 

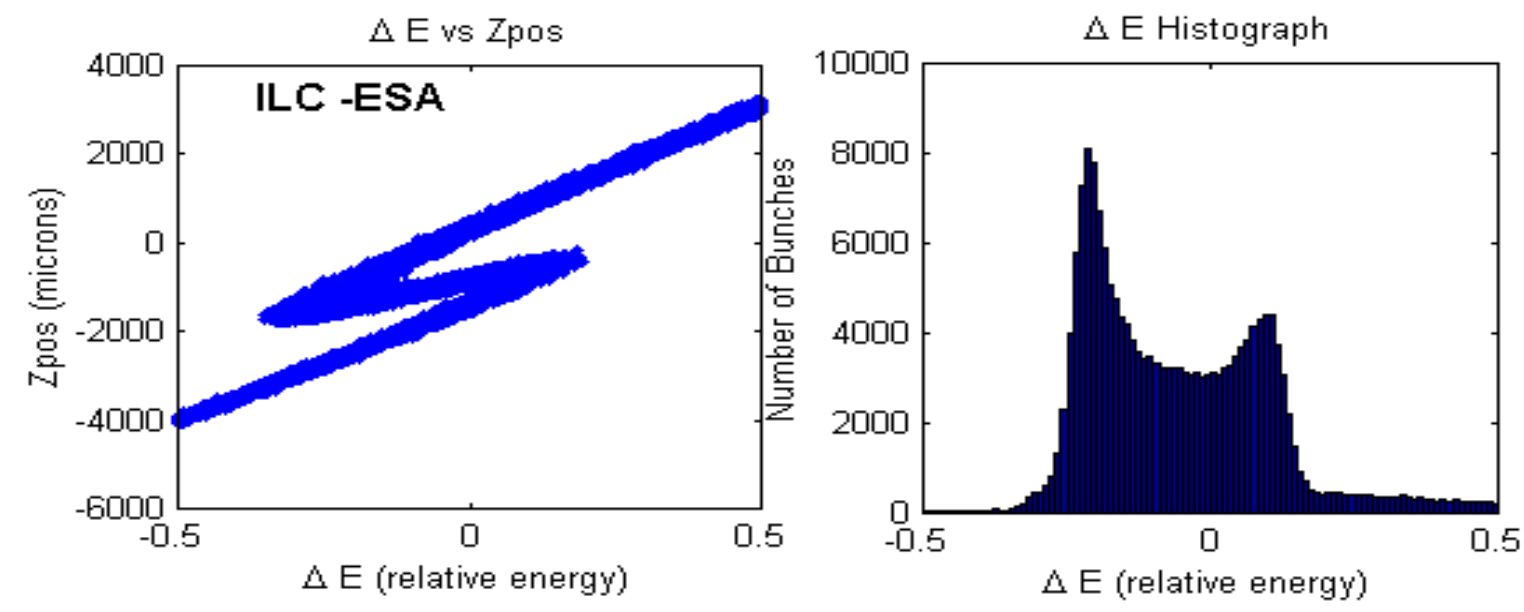

Figure 12. Example of histogram and plot generated from Program 1 . The figure on the left show the relationship between the Zpos and relative energy. The figure on the right shows a histogram of the relative energy.

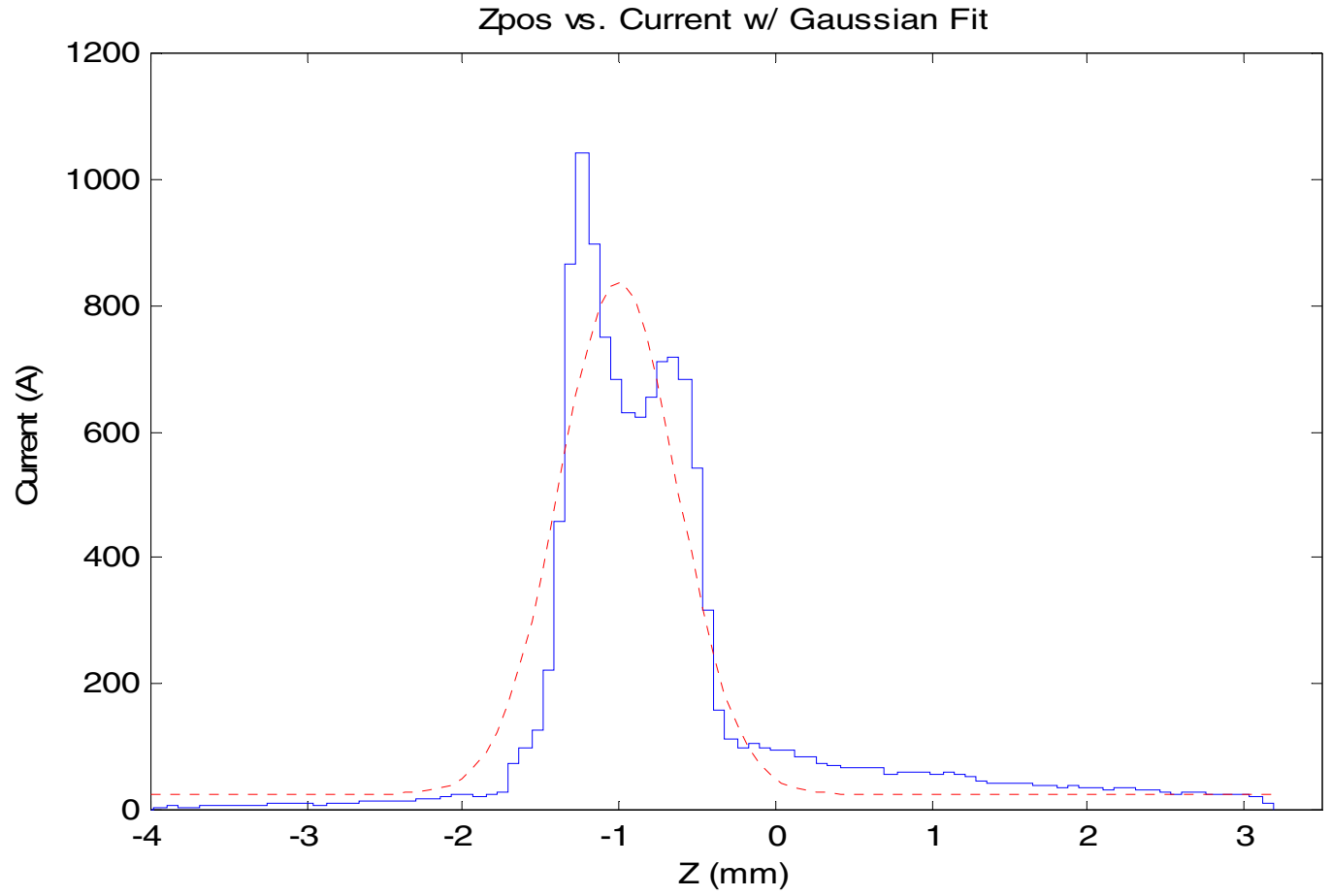

Figure 13. Example of plot generated from Program 3. The plot show the relation ship between the Zpos and Current. The red dotted line is a Gaussian fit for the relationship between the Zpos and Current 
